\title{
Research on Metal Nanostructure Building Based on Nanoimprint Lithography
}

\author{
Minqi Yin, Hongwen Sun \\ College of Internet of Things Engineering, HohaiUniversity, Changzhou, \\ 213022, China
}

\begin{abstract}
We use the nanoimprint lithography to construct nanostructure on the surface of double metal $\mathrm{Au}$ electrode. Through the analysis of the influence of different processing parameters on the machining dimensions, we can arbitrarily control the structure size of the nano-pattern. By natural oxidation in air and selection of the appropriate parameters, the nanostructured metals are obtained. More importantly, we have successfully fabricated the inverse structure of the gold nano hemisphere array on the flexible substrate.
\end{abstract}

Keywords:metal nanostructure, structure building, nanoimprint lithography

\section{Introduction of nanoimprint lithography}

Chinese American scientist Zhou Yu of Princeton University nano structure laboratory in 1995 for the first time put forward a new method of nano copy graphics, to showcase the technology for fabrication of nanostructured template based model. Nano imprint technology. Nano imprint refers to the nano structure template surface in polymer film, in certain conditions such as temperature and pressure, the polymer gradually filled into nano template in the cavity for curing polymer is formed, and the polymer template will be stripped, a method to implement the transfer of the nano structure. Different from the traditional principle of nano imprint lithography, is a method of structure transfer using nano imprint template structure in the transfer process, the structure size is determined by the template size, completely unaffected by the wavelength of light, it is not affected by the optical wavelength diffraction limit on the construction of structure, so show unique advantages of super high resolution, high yield, low 
cost and suitable for industrial applications. Nano imprint processing due to its advantages of low cost and high efficiency, simple operation, can be widely used in solar cells, sensors, data storage, biomedical and other fields, people pay more and more attention. However, nano imprint lithography to a large area of industrial production, but also face some problems. Nano imprint lithography technology is still in the development period. There will be a decisive advantage in the future competition.

\section{Experiment of metal nanostructure building}

Nanoimprint lithography system experiment uses the Seiko production of SPA400 , with the tip of the silicon nitride etching is of high hardness, needle tip arc radius of curvature is less than $20 \mathrm{~nm}$, the cantilever elastic modulus is $2 \mathrm{~N} / \mathrm{m}$, processing and imaging using the same probe, and $\mathrm{C}$ - nano imprint is tested using a gold-plated probe. The working mode of the utility model is a contact type, and the etching is carried out in an atmospheric room temperature environment. Nano imprint lithography to achieve a total of two ways, one is in the imaging mode, and the other is to run through the program control. In the imaging mode, the graphics processing only for the simple square area, and probes in the characterization for dot matrix scanning, effect of scanner drift on the structure after processing is more serious; and the program control system has a Vector Scan by nano imprint, in software command, can be based on the graphics processing of the preset the probe moving path, direction and corresponding processing parameters, so as to obtain the nano structure we want, because the probe processing by the restrictions, in the next image, graphics is neat, the scanner drift effect is not obvious, so the processing of this experiment in the etching program to achieve. First of all, nano imprint tip load does not, the images of the samples were obtained when processing the image of a sample surface, and select the smaller surface roughness region; after the start, the etching procedure, in the program command, preset the following parameters: the tip of the load path, probe and probe the size and number of carved rate. In the imaging mode is switched to the etching mode when the probe is first moved to the center position of the processing area, and then carved the first line according to the order, at the end point of the first line as the initial point of the next line of the probe position between the two lines, need to set up a system to wait for orders have enough time to finish it in time. The etching rate relatively large is very necessary.

Surface-enhanced RamanScattering (SERS) is the phenomenon of the Raman scattering spectra of the molecules on the surface of the metal surface under the excitation of the external electromagnetic field. Surface enhanced Raman scattering enhancement factor EF is used to describe the enhancement of Surface Raman scattering. Compared with the conventional Raman scattering, the surface enhanced Raman scattering spectrum has a higher sensitivity to Raman scattering. SERS is a powerful spectroscopic technique with high detection sensitivity and can detect very low concentrations of analyses. Therefore, in the identification of organic dyes and pigments, pigment has a wide range of applications. At present, 
the materials reported in the literature can be used as SERS active substrates, such as silver, gold, copper and other currency metals and lithium, sodium, potassium and other alkali metals and some metal oxides and semiconductor materials. In the field of chemical and biological analysis, the metal nano materials, or nanowires, show high sensitivity. This results in the uneven distribution of the target molecule. In order to solve this problem, it is necessary to prepare the SERS substrate which can be precisely controlled. In practical SERS applications, many researchers used different lithography techniques to prepare periodic metal nanostructures, including electron beam lithography and soft lithography. However, the simple, large area, highly ordered preparation of metal nano array structure is a major problem to be solved. In this regard, nano ball etching technology and colloidal plate printing technology, nano imprint technology, whichcan be used with simple operation, low cost preparation of metal nanostructure arrays. Metal nano structure array slot size, sharpness and roughness of geometrical parameters are very important, can affect the Raman signal intensity. Because the electromagnetic field enhancement occurs mainly in the middle of the small metal gap, the lightning rod effect occurs at the sharp edge.

At the end of a cycle, the probe back to the initial position, the next cycle of the specified number of times to complete the entire processing command. Finally, the tip load is removed, and then the image is scanned in the scanning mode, and the image is processed by nano imprint lithography. With the preparation of $\mathrm{Ag}$ nano hemisphere array operation is basically the same, the same in PMMA stamping structure with a hemispherical surface, evaporation coating machine by magnetron sputtering magnetron sputtering a layer of gold film, obtained is $\mathrm{Au}$ (nano hemisphere diameter of about $550 \mathrm{~nm}$ ) array structure. We have sputtered $\mathrm{Au}$ films at different times of the six corners of the dense Au array of nano hemisphere arrays of SEM. The results show that the preparation of gold nano hemisphere array, a large area of highly ordered. In order to study the optical properties of the prepared Au nano hemisphere arrays, we tested the UV-visa on the hemispherical array with different time of $\mathrm{Au}$, as shown in figure 2-7. Two absorption peaks have appeared. With the increase of the sputtering time of $\mathrm{Au}$ film, the noise in the absorption spectrum is relatively strong. In subsequent experiments, we selected Au films with sputtering time of $150 \mathrm{~s}$.

\section{Results and discussion}

We set up 5 lines in the program instructions from right to left load increases ( 10 , $100,200,300,400 \mathrm{nN}$ ), the command cycle times is 10 , the rate is $5 \mathrm{~m} / \mathrm{s}$ mobile probe. When the load changes from $10 \mathrm{nN}$ to $400 \mathrm{nN}$, the depth of the groove are respectively increased from $1 \mathrm{~nm}$ to $4 \mathrm{~nm}$, from the corresponding profile analysis can be very easy to see this trend, with the increase of force, groove depth and width increases, but not obvious in small notch under load. For the different shapes of the probe at the rate of the formation of the graphics and the corresponding profile analysis, the 5 lines from right to left rate gradually 
increased, the size of the probe on a constant load of $400 \mathrm{nN}$, the number of cycles was 10 times. When the rate ranges from $0.1 \mathrm{~m} / \mathrm{s}$ to $10 \mathrm{~m} / \mathrm{s}$, the depth of the groove respectively by $4.7 \mathrm{~nm}$ decreases to about $2 \mathrm{~nm}$, that is, the extension of the interaction time between the probe and the sample will increase the depth and width of the etching area. This is due to the lower the rate of the probe on the sample in a long time, will produce a relatively large pressure, the pressure can make the probe easier to characterize, resulting in a deeper groove. We should also analyze the shapes of different cycles and the corresponding profiles. The 5 line from left to right, the number were $1,10,20,30,40$, the probe load constant is $400 \mathrm{nN}$, the rate was 5 mobile probe $\mathrm{m} / \mathrm{s}$, the depth of the groove are respectively increased from $1 \mathrm{~nm}$ to $3.7 \mathrm{~nm}$, that is, with the increase of the number of orders, the time when the probe is applied to the sample increases and the depth increases linearly. The shape of the groove formed by processing is $\mathrm{V}$, which is caused by the shape of the probe. The above results indicate that the probe load and load duration in the sample are the main factors influencing the etching results. The larger the load is, the longer the time, machining depth and width increases. However, the probe rate is too small, and the processing speed is slow.

By using RIE, the PMMA nano hemisphere arrays were etched, and the Au nano films with different morphologies were obtained by etching the Au film of $150 \mathrm{~s}$ after different etching time, respectively. With the increase of etching time of RIE, the diameter of PMMA nanospheres is smaller. When the RIE etching time is $5 \mathrm{~s}$, the spacing between the hemisphere and hemisphere of a few nanometers; the etching time is $20 \mathrm{~s}$, the distance between the hemisphere and hemisphere was 15 $\mathrm{nm}$; the etching time is $40 \mathrm{~s}$, the distance between the hemisphere and hemisphere was $40 \mathrm{~nm}$; the etching time is $60 \mathrm{~s}$, the distance between the hemisphere and hemisphere $80 \mathrm{~nm}$. With the increase of the etching time of RIE, not only the space between the nano hemispheres increased, but also the roughness of the surface of the nano sphere increased. Therefore, we can control the RIE etching of PMMA nano hemisphere in different time, to change the structure parameters, such as: Au nano hemisphere array spacing between Nano hemisphere diameter, surface roughness and nano nano hemisphere hemisphere. UV-visa tests were performed for different Au hemispherical arrays obtained by adjusting etching time. There are two absorption peaks in the Au hemisphere arrays, which are about $610 \mathrm{~nm}$ and $900 \mathrm{~nm}$, respectively.

With the increase of etching time, the absorption peak at $610 \mathrm{~nm}$ has a red shift. With the increase of etching time, the peak shifts from $610 \mathrm{~nm}$ to about $660 \mathrm{~nm}$. However, with the increase of etching time, the peak position at $900 \mathrm{~nm}$ is almost no shift. Our analysis shows that there is a transmission peak at about $900 \mathrm{~nm}$, which is caused by the incident of the gold nano ordered array as a twodimensional diffraction grating, and the incident photon is converted to SP wave. Although the etching time is changed, the period of the gold nano hemisphere array does not change, so the peak position of $900 \mathrm{~nm}$ does not move. On the surface of the prepared Au nano hemisphere array, a layer of PDMS was poured, 
solidified and peeled off to obtain the Au nano bowl array on the PDMS substrate. We can see that the nano bowl array structure is obtained by the nano hemisphere array structure, we generally call the nano hemisphere array structure is positive structure, nano bowl array structure for negative type structure. The dimensions of negative structures are generally determined by positive structure. The test results show that the photo catalytic efficiency, Zinc Oxide nanorods have significantly improved on the degradation rate of methylene blue, but the gold nano structure is not improved. We did not achieve the desired effect. The gold nano bowl array on the flexible substrate is also highly ordered in large area. The diameter of the nano bowl is about $460 \mathrm{~nm}$. We can see from the illustration that the gold nano bowl array on the flexible substrate is observed in natural light, which is very easy to bend and easy to recover. In the experiment, we also try to use transparent tape as a flexible substrate, the results show that the same can be obtained large area ordered Au nano bowl array, does not require curing molding, so the operation is simpler.

\section{Conclusion}

The patterned metal nanostructures have been widely used inSurface Plasmon Resonce (SPR) sensors and solar cells. In this paper, a large area and ordered patterned metal nano-array structure was fabricated by using Lithography (Nanoimprint) and NIL magnetron sputtering deposition technique. Surfaceenhanced Raman scattering (SERS) tests were performed on the prepared metal nano hemisphere arrays, which have strong SERS characteristics.

\section{References}

[1]Chen Jiangang, Wei Pei, Chen Jiefeng, Zhao Zhixin, He Yajuan, Research and development of nanoimprint lithography technology, Journal of Shaanxi University of Technology (Natural Science Edition), 29(5), pp. 1-5+9, 2013.

[2] Wang Jinhe, FeiLicheng, Song Zhitang, Zhang Jing, Zhou Weimin, Zhang Jianping, LatestProgressinNano-ImprintLithography, Micronanoelectronic Technology, 47(12), pp. 722-730, 2010.

[3]Peng Jing, XuZhimou, Wu Xiaofeng, Sun Tangyou, A study of LED with surface photonic crystalstructure fabricated by the nanoimprint lithography, ActaPhysicaSinica, 62(3), pp. 1-7, 2013.

[4] Zhao Jianyi, Chen Xin, Zhou Ning, Cao Mingde, Huang Xiaodong, Liu Wen, Reliability Studyof DFB Laser Fabricated by Nanoimprint Technology, ActaOpticaSinica, 34(2), pp. 1-7, 2014. 